\title{
Extended states in systems with odd-rank transfer matrices
}

\author{
S D Wang ${ }^{1,3}$, Zhou-Zhou Sun ${ }^{1}$, Gang Xiong ${ }^{1,2}$, S Yin ${ }^{1}$ and X R Wang ${ }^{1}$ \\ ${ }^{1}$ Department of Physics, Hong Kong University of Science and Technology, Hong Kong, \\ People's Republic of China \\ 2 Department of Physics, Beijing Normal University, Beijing 100875, China
}

Received 1 May 2003, in final form 24 October 2003

Published 9 January 2004

Online at stacks.iop.org/JPhysA/37/1337 (DOI: 10.1088/0305-4470/37/4/019)

\begin{abstract}
The localization properties are investigated within a transfer matrix formulation. We find that there may exist one extended eigenstate when the rank of the transfer matrix is odd. An edge state in a quantum Hall system is such an example. It is a chiral state that can always carry a current.
\end{abstract}

PACS numbers: 03.65.-w, 72.10.Bg, 73.20.Jc

\begin{abstract}
A non-interacting Schrödinger equation can be formulated into a transfer matrix form. This method has been used to numerically study Anderson localization and to investigate electron transport properties of mesoscopic systems [1-4]. Anderson et al [5] used this method to study the one-dimensional localization problem. Shapiro generalized the formulation into a high dimensional system [6]. In the Anderson localization model, an electron in a channel can move in two opposite directions due to the time reversal symmetry. The rank of an $m$-channel transfer matrix is $2 m$, an even number, because there are two modes on each channel. The transfer matrix method is also used to investigate transport properties of a two-dimensional electron gas under a strong magnetic field, a quantum Hall (QH) system in which the time reversal symmetry is broken. According to a semiclassical model, an electronic state in a strong magnetic field and a smooth potential can be decomposed into a rapid cyclotron motion and a slow drifting motion of its guiding centre. The direction of the drifting motion is unidirectional, i.e., electrons are in chiral states [7]. One can use a so-called Chalker-Coddington (CC) network model to investigate a $\mathrm{QH}$ system [8]. In this model, electrons on each link can move in only one direction. Thus there is only one mode on each channel. Consequently, the rank of the transfer matrix in the CC model, which is the number of independent modes, may be odd because there is no restriction on the channel number. This is different from the transfer matrix in the Anderson localization model, whose

3 Current address: Institut für Theoretische Physik, Universitat Regensburg, 93040 Regensburg, Germany.
\end{abstract}


rank is always even. According to the scaling argument of localization [9], all electrons in a two-dimensional system are localized in the absence of a magnetic field. However, there exist extended states in a QH system, e.g. edge states. They are chiral states and can always carry a current. Because of these distinguishable outcomes of the two cases, one may wonder whether localization properties of a system depend on the rank of its transfer matrix.

In this paper, we investigate how the rank of a transfer matrix affects electron localization. We find that there may exist at least one edge-state-like eigenstate when the transfer matrix rank is odd. This kind of extended state can always carry a current because of its chiral nature. For a transfer matrix with even rank, such a definite result does not exist.

For simplicity, we consider a transfer matrix of a strap system with $N$ modes. Here, we assume that an electron in a mode moves in a definite direction and we call this direction the direction of the mode. Therefore, a channel in which electrons can move in two directions is separated into two unidirectional modes while a channel in which electrons can move in only one direction is represented by one unidirectional mode. A transfer matrix in the Anderson localization model has the same numbers of modes with opposite directions and its rank $N$ is always even. And a transfer matrix in the CC model may have different numbers of modes with opposite directions and its rank $N$ can be either even or odd. In the vector space spanned by a set of $N$ linearly independent eigenmodes, $|i\rangle(i=1, \ldots, N)$, a vector variable of the wavefunction at the $x$ th column can be introduced as

$$
\boldsymbol{\Phi}_{N}(x)=\left(\begin{array}{c}
\phi_{1}(x) \\
\vdots \\
\phi_{N}(x)
\end{array}\right)
$$

where the $i$ th component, $\phi_{i}(x)$, is the probability amplitude in basis vector $|i\rangle$. The transfer matrix $\hat{M}_{N}(L)$ of the system is defined as

$$
\boldsymbol{\Phi}_{N}(L)=\hat{M}_{N}(L) \Phi_{N}(0)
$$

where $\boldsymbol{\Phi}_{N}(0)$ and $\boldsymbol{\Phi}_{N}(L)$ are the wavefunctions on two sides of the system, $x=0$ and $x=L$, respectively. Therefore, the transfer matrix relates the wavefunction on two different sides, i.e., one can obtain the wavefunction of a system on one side from that on the other side. The form of the transfer matrix depends generally on the way we choose these two sides. A system with transfer matrix $\hat{M}_{N}(L)$ with rank $N$ is schematically shown in figure 1. A transfer matrix $\hat{M}_{N}(L)$ relates wavefunctions $\boldsymbol{\Phi}_{N}(0)$ and $\boldsymbol{\Phi}_{N}(L)$. Lines represent propagating modes. An electron in a mode moves along one direction indicated by an arrow.

In order to obtain the rank dependence properties of a transfer matrix $\hat{M}_{N}(L)$, we first prove four lemmata. It should be pointed out that many results in these lemmata are already known for the symplectic transfer matrices used in the Anderson localization [2-4, 10]. The reason that we present them here is to understand our central result in the theorem below. We define a current operator $\hat{J}_{N}(L)$. Its elements are given as

$$
\left(\hat{J}_{N}(L)\right)_{i j}=v_{i} \delta_{i j}
$$

where

$$
\delta_{i j}= \begin{cases}1 & \text { if } \quad i=j \\ 0 & \text { if } \quad i \neq j\end{cases}
$$

Also $v_{i}$ is the velocity of an electron in mode $i$. We define that $v_{i}=1$ if the direction of the mode is from left to right and that $v_{i}=-1$ if the mode direction is from right to left. The expectation value of $\hat{J}_{N}(L)$ is the net current carried by the corresponding wavefunction. 


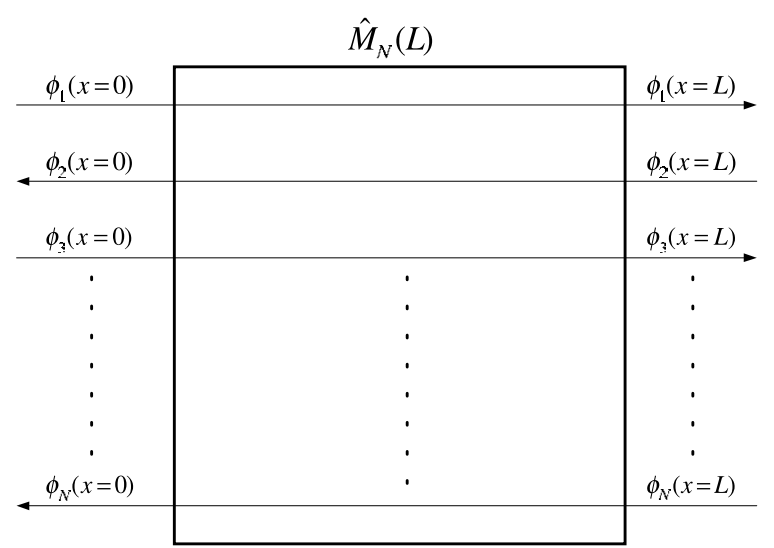

Figure 1. A transfer matrix $\hat{M}_{N}(L)$ with rank $N$ relates wavefunctions $\Phi_{N}(0)$ and $\Phi_{N}(L)$ of a system. The square box represents the transfer matrix $\hat{M}_{N}(L)$ of the system. $\Phi_{N}(0)$ and $\Phi_{N}(L)$ are the wavefunctions on the left and right sides. Lines represent propagating modes and arrows denote the directions of the modes.

Lemma 1. A transfer matrix $\hat{M}_{N}(L)$ and the current operator $\hat{J}_{N}(L)$ satisfy the relations

$$
\hat{M}_{N}^{\dagger}(L) \hat{J}_{N}(L) \hat{M}_{N}(L)=\hat{J}_{N}(L)
$$

and

$$
\hat{M}_{N}(L) \hat{J}_{N}(L) \hat{M}_{N}^{\dagger}(L)=\hat{J}_{N}(L)
$$

Proof. Because of the continuity of current in the stationary state, the currents flowing into and out of the system, that is, the currents at $x=0$ and $x=L$ are the same and we have

$$
\boldsymbol{\Phi}_{N}^{\dagger}(0) \hat{J}_{N}(L) \Phi_{N}(0)=\Phi_{N}^{\dagger}(L) \hat{J}_{N}(L) \Phi_{N}(L) .
$$

By using equation (2), we have

$$
\boldsymbol{\Phi}_{N}^{\dagger}(0) \hat{J}_{N}(L) \Phi_{N}(0)=\Phi_{N}^{\dagger}(0) \hat{M}_{N}^{\dagger}(L) \hat{J}_{N}(L) \hat{M}_{N}(L) \Phi_{N}(0) .
$$

Because the above relation must hold for any wavefunction $\Phi_{N}(0)$, we have

$$
\hat{M}_{N}^{\dagger}(L) \hat{J}_{N}(L) \hat{M}_{N}(L)=\hat{J}_{N}(L) \text {. }
$$

By taking the inverse of $\hat{J}_{N}(L)$, we have

$$
\hat{M}_{N}^{-1}(L) \hat{J}_{N}^{-1}(L)\left(\hat{M}_{N}^{\dagger}\right)^{-1}(L)=\hat{J}_{N}^{-1}(L) .
$$

According to the definition of the current operator $\hat{J}_{N}(L)$, equation $(3), \hat{J}_{N}^{-1}(L)=\hat{J}_{N}(L)$. After multiplying $\hat{M}_{N}(L)$ from the left and $\hat{M}_{N}^{\dagger}(L)$ from the right, we have

$$
\hat{M}_{N}(L) \hat{J}_{N}(L) \hat{M}_{N}^{\dagger}(L)=\hat{J}_{N}(L) .
$$

Therefore, we prove the lemma.

Lemma 2. If $\lambda$ is an eigenvalue of a transfer matrix $\hat{M}_{N}(L), 1 / \lambda^{*}$ is also its eigenvalue.

Proof. Let $\Psi_{N}$ be an eigenstate of $\hat{M}_{N}(L)$ with eigenvalue $\lambda$. We have

$$
\hat{M}_{N}(L) \Psi_{N}=\lambda \Psi_{N}
$$


where the eigenstate $\boldsymbol{\Psi}_{N}$ is defined as

$$
\boldsymbol{\Psi}_{N}(x)=\left(\begin{array}{c}
\psi_{1}(x) \\
\vdots \\
\psi_{N}(x)
\end{array}\right) .
$$

By multiplying equation (6) with $\hat{M}_{N}^{\dagger}(L) \hat{J}_{N}(L)$ from left, we have

$$
\hat{M}_{N}^{\dagger}(L) \hat{J}_{N}(L) \hat{M}_{N}(L) \Psi_{N}=\lambda \hat{M}_{N}^{\dagger}(L) \hat{J}_{N}(L) \Psi_{N} .
$$

By using equation 5(a), we have

$$
\hat{M}_{N}^{\dagger}(L)\left(\hat{J}_{N}(L) \Psi_{N}\right)=\lambda^{-1}\left(\hat{J}_{N}(L) \Psi_{N}\right) .
$$

Therefore $\left(\hat{J}_{N}(L) \Psi_{N}\right)$ is an eigenstate of $\hat{M}_{N}^{\dagger}(L)$ with eigenvalue $1 / \lambda$. Because $\hat{M}_{N}^{\dagger}(L)=$ $\left(\hat{M}_{N}^{T}(L)\right)^{*}, 1 / \lambda^{*}$ is an eigenvalue of the transpose $\hat{M}_{N}^{T}(L)$. And the eigenvalues of a matrix and its transpose are the same because $\operatorname{det}\left(M^{T}-\lambda I\right)=\operatorname{det}(M-\lambda I)$. Therefore, $1 / \lambda^{*}$ is an eigenvalue of $\hat{M}_{N}(L)$.

Lemma 3. The rank $N$ of a transfer matrix $\hat{M}_{N}(L)$ of a system with time reversal symmetry is an even number and the numbers of modes with opposite directions are equal.

Proof. In a system with time reversal symmetry, the complex conjugate of its wavefunction $\boldsymbol{\Phi}_{N}$ is also its wavefunction. We have the similar relation as equation (2),

$$
\boldsymbol{\Phi}_{N}^{*}(L)=\hat{M}_{N}(L) \Phi_{N}^{*}(0) .
$$

Suppose that there are $m$ modes whose directions are from left to right and the corresponding probability amplitudes are $\phi_{i}(x), i=1, \ldots, m$. And there are $(N-m)$ modes whose directions are from right to left and the corresponding probability amplitudes are $\phi_{i}(x), i=$ $(m+1), \ldots, N$. We separate the wavefunction $\boldsymbol{\Phi}_{N}$ into two parts, $\boldsymbol{\Phi}_{+}(x)$ and $\boldsymbol{\Phi}_{-}(x) . \boldsymbol{\Phi}_{+}(x)$ contains all modes in which electrons move from left to right while $\Phi_{-}(x)$ contains the modes in which electrons move from right to left. These two parts of the wavefunction are defined as

$$
\boldsymbol{\Phi}_{+}(x)=\left(\begin{array}{c}
\phi_{1}(x) \\
\vdots \\
\phi_{m}(x)
\end{array}\right) \quad \boldsymbol{\Phi}_{-}(x)=\left(\begin{array}{c}
\phi_{m+1}(x) \\
\vdots \\
\phi_{N}(x)
\end{array}\right) .
$$

According to equation (2), we have

$$
\left(\begin{array}{l}
\boldsymbol{\Phi}_{+}(L) \\
\boldsymbol{\Phi}_{-}(L)
\end{array}\right)=\hat{M}_{N}(L)\left(\begin{array}{l}
\boldsymbol{\Phi}_{+}(0) \\
\boldsymbol{\Phi}_{-}(0)
\end{array}\right) .
$$

By using equation (9), we also have

$$
\left(\begin{array}{l}
\boldsymbol{\Phi}_{-}^{*}(L) \\
\boldsymbol{\Phi}_{+}^{*}(L)
\end{array}\right)=\hat{M}_{N}(L)\left(\begin{array}{l}
\boldsymbol{\Phi}_{-}^{*}(0) \\
\boldsymbol{\Phi}_{+}^{*}(0)
\end{array}\right)
$$

where the direction of $\boldsymbol{\Phi}_{-}^{*}(x)$ is from left to right while the direction of $\boldsymbol{\Phi}_{+}^{*}(x)$ is from right to left. In equation (12), there are $(N-m)$ modes with direction from left to right in $\boldsymbol{\Phi}_{-}^{*}(x)$ and $m$ modes from right to left in $\boldsymbol{\Phi}_{+}^{*}(x)$. Because it is the same transfer matrix $\hat{M}_{N}(L)$ in two equations, we have $N=2 m$, that is, the rank of the transfer matrix of a system with time reversal symmetry is even and the numbers of modes with opposite directions are equal.

Lemma 4. If $\lambda$ is an eigenvalue of the transfer matrix $\hat{M}_{N}(L)$ of a system with time reversal symmetry, $1 / \lambda^{*}, \lambda^{*}$, and $1 / \lambda$ are its eigenvalues, too. 
Proof. We define an operator $\hat{R}_{N}(L)$ of a system with time reversal symmetry. Its elements are given as

$$
\left(\hat{R}_{N}(L)\right)_{i j}= \begin{cases}\delta_{(i+N / 2) j} & \text { if } \quad i=1, \ldots, N / 2, \\ \delta_{(i-N / 2) j} & \text { if } \quad i=N / 2+1, \ldots, N\end{cases}
$$

where $\delta_{i j}$ is defined in equation (4). $N$ is the rank of a transfer matrix of a system with time reversal symmetry and it is an even number according to lemma 3 . The operator $\hat{R}_{N}(L)$ has the property

$$
\hat{R}_{N}(L) \hat{R}_{N}(L)=1
$$

And we have the relation

$$
\hat{R}_{N}(L)\left(\begin{array}{l}
\Phi_{-}(x) \\
\Phi_{+}(x)
\end{array}\right)=\left(\begin{array}{l}
\Phi_{+}(x) \\
\Phi_{-}(x)
\end{array}\right) .
$$

By taking the complex conjugate of equation (12), we have

$$
\left(\begin{array}{l}
\boldsymbol{\Phi}_{-}(L) \\
\boldsymbol{\Phi}_{+}(L)
\end{array}\right)=\hat{M}_{N}^{*}(L)\left(\begin{array}{l}
\boldsymbol{\Phi}_{-}(0) \\
\boldsymbol{\Phi}_{+}(0)
\end{array}\right) .
$$

After multiplying the operator $\hat{R}_{N}(L)$ from the left, we have

$$
\left(\begin{array}{l}
\boldsymbol{\Phi}_{+}(L) \\
\boldsymbol{\Phi}_{-}(L)
\end{array}\right)=\hat{R}_{N}(L) \hat{M}_{N}^{*}(L) \hat{R}_{N}(L)\left(\begin{array}{l}
\boldsymbol{\Phi}_{+}(0) \\
\boldsymbol{\Phi}_{-}(0)
\end{array}\right)
$$

where we use equations (14) and (15). Because $\Phi$ is an arbitrary wavefunction, equations (11) and (17) must be equivalent. Therefore, we have

$$
\hat{M}_{N}(L)=\hat{R}_{N}(L) \hat{M}_{N}^{*}(L) \hat{R}_{N}(L) .
$$

By multiplying $\hat{R}_{N}(L)$ from the left and using equation (14), we have

$$
\hat{R}_{N}(L) \hat{M}_{N}(L)=\hat{M}_{N}^{*}(L) \hat{R}_{N}(L) .
$$

By multiplying $\hat{R}_{N}(L)$ to equation (6) from left and using equation (18), we have

$$
\hat{M}_{N}^{*}(L)\left(\hat{R}_{N} \Psi_{N}\right)=\lambda\left(\hat{R}_{N} \Psi_{N}\right) \text {. }
$$

By taking the complex conjugate, we have

$$
\hat{M}_{N}(L)\left(\hat{R}_{N} \Psi_{N}\right)^{*}=\lambda^{*}\left(\hat{R}_{N} \Psi_{N}\right)^{*}
$$

Hence $\lambda^{*}$ is also an eigenvalue of $\hat{M}_{N}(L)$. According to lemma $2,1 / \lambda^{*}$ and and $1 / \lambda$ are also eigenvalues of $\hat{M}_{N}(L)$.

Now, we shall prove the rank dependence properties of eigenstates of a transfer matrix $\hat{M}_{N}(L)$, the central result of this paper.

Theorem 1. Let $\Psi_{N}$ be an eigenstate of $\hat{M}_{N}(L)$ with eigenvalue $\lambda$. If $|\lambda| \neq 1, \Psi_{N}$ is a localized state that cannot carry a current through the system. If $\Psi_{N}$ is an extended state, its corresponding eigenvalue must satisfy $|\lambda|=1$. That is,

$$
\begin{cases}\boldsymbol{\Psi}_{N}^{\dagger} \hat{J}_{N}(L) \boldsymbol{\Psi}_{N}=0 & \text { if } \quad|\lambda| \neq 1, \\ |\lambda|=1 & \text { if } \quad \boldsymbol{\Psi}_{N}^{\dagger} \hat{J}_{N}(L) \boldsymbol{\Psi}_{N} \neq 0\end{cases}
$$

where $\hat{J}_{N}(L)$ is the current operator defined in equation (3). Furthermore, there is at least one eigenstate with its eigenvalue, $|\lambda|=1$, when the rank of the transfer matrix is odd.

Proof. According to equations 5(a) and (6), we have $\boldsymbol{\Psi}_{N}^{\dagger} \hat{J}_{N}(L) \boldsymbol{\Psi}_{N}=\boldsymbol{\Psi}_{N}^{\dagger} \hat{M}_{N}^{\dagger}(L) \hat{J}_{N}(L) \hat{M}_{N}(L) \boldsymbol{\Psi}_{N}=\boldsymbol{\Psi}_{N}^{\dagger} \lambda^{*} \hat{J}_{N}(L) \lambda \boldsymbol{\Psi}_{N}=|\lambda|^{2} \boldsymbol{\Psi}_{N}^{\dagger} \hat{J}_{N}(L) \boldsymbol{\Psi}_{N}$. 
Thus, we have

$$
\left(|\lambda|^{2}-1\right) \Psi_{N}^{\dagger} \hat{J}_{N}(L) \Psi_{N}=0
$$

that is

$$
\left\{\begin{array}{lll}
\boldsymbol{\Psi}_{N}^{\dagger} \hat{J}_{N}(L) \boldsymbol{\Psi}_{N}=0 & \text { if } & |\lambda| \neq 1, \\
|\lambda|=1 & \text { if } & \boldsymbol{\Psi}_{N}^{\dagger} \hat{J}_{N}(L) \boldsymbol{\Psi}_{N} \neq 0 .
\end{array}\right.
$$

$\Psi_{N}^{\dagger} \hat{J}_{N}(L) \Psi_{N}$ describes the current carried by the eigenstate $\boldsymbol{\Psi}_{N}$. Therefore the eigenstates with eigenvalues $|\lambda| \neq 1$ are localized states. From lemma 2 , we know that both $\lambda$ and $1 / \lambda^{*}$ are eigenvalues of the transfer matrix $\hat{M}_{N}(L)$. If $\hat{M}_{N}(L)$ has an odd rank, there exists at least one eigenvalue such that $\lambda=1 / \lambda^{*}$, that is, $|\lambda|^{2}=1$. Therefore, there is at least one eigenstate with its eigenvalue $|\lambda|=1$ of a transfer matrix with odd rank. Thus the state must be either extended or critical [10]. However, a transfer matrix with even rank does not have this property. If an eigenstate is extended, it can carry a current since the expectation value of current operator $\hat{J}_{N}(L)$ in this state may not be zero.

The transfer matrix described in this paper is usually called a 'global' transfer matrix. Theorem 1 shows that the structure of the local transfer matrix is unimportant. Theorem 1 has indeed been verified in some recent publications [11, 12]. It was shown that at least one eigenstate is extended when the transfer matrix rank is odd. The difference between the Anderson localization model and the $\mathrm{CC}$ model can be understood from theorem 1. Because the rank of a transfer matrix in a CC model may be an odd number, there may exist an extended state. However, the rank of a transfer matrix in an Anderson localization model is always even, there may be no extended state in this system. In fact all electrons are localized in a two-dimensional system, which can be described by an Anderson localization model. As the size of a system becomes larger and larger, the number of states needed to describe the system becomes larger and larger. When we use a transfer matrix $\hat{M}_{N}(L)$ to describe a high dimensional system, we must let the system size, $L \rightarrow \infty$. Under this limit, the number of eigenstates of the transfer matrix $\hat{M}_{N}(L)$ tends to infinity as well. We shall ask whether the extended state in a transfer matrix described by theorem 1, whose number is finite, can really play an important role. As we know that properties of a usual system are determined by the average of large numbers of available states and the effect of one or finite number states can be safely ignored. However, there exist systems whose properties are determined by only a few states. For example, one edge state of a QH system causes one Hall plateau and all properties of a superconductor depend on its ground state below the transition temperature. Therefore, we can use theorem 1 to obtain some general properties of this kind of system. The extended state described by theorem 1 always carries a current. In a transfer matrix of a system with time reversal symmetry, there may exist some other kinds of extended eigenstates, which always appear in pair according to lemma 4. The pair of eigenstates with the eigenvalues $\lambda$ and $\lambda^{*}$ have opposite directions. For example, in a system with time reversal symmetry, its energy eigenstates are momentum degenerate, that is, two eigenstates with opposite momenta have the same energies. In this system, an extended energy eigenstate cannot carry a net current when it lies below the chemical potentials of source and drain because electrons can tunnel through this state in two opposite directions. However, if an eigenstate is extended and is described by theorem 1, it does not appear in pairs and electrons can move in only one direction in this state. Therefore this state can carry a net current due to its chiral nature. The edge states in a QH system are this kind of state. As shown in figure 2, edge states on the same edges of a sample carry currents in the same direction while edge states on two opposite edges carry currents in opposite directions. An edge state can carry a current even when they 


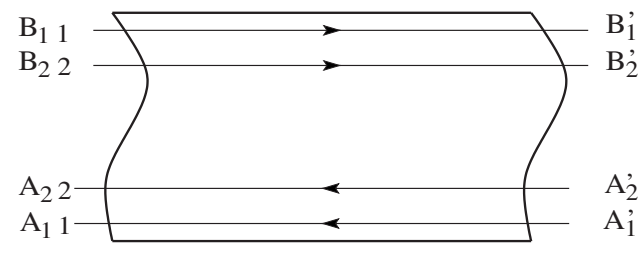

Figure 2. A QH system containing two edge states. The edge states on the top carry current from left to right while those at bottom carry current in the opposite direction, as indicated by arrows.

lie below the chemical potential of source and drain, which causes a Hall plateau in a QH system. Therefore, an edge state in a $\mathrm{QH}$ system is an example of the extended state of an odd rank transfer matrix.

\section{Acknowledgments}

This work was supported by grants from the Research Grant Council of HKSAR, China (project nos HKUST6153/99P, and HKUST6149/00P).

\section{References}

[1] Landauer R 1970 Phil. Mag. 21863

[2] Kramer B and MacKinnon A 1993 Rep. Prog. Phys. 561469

[3] Belitz D and Kirkpatrick T R 1994 Rev. Mod. Phys. 66261

[4] Römer R A and Schreiber M 2002 Preprint cond-mat/0212569

[5] Anderson P W, Thouless D J, Abrahams E and Fisher D S 1980 Phys. Rev. B 223519

[6] Shapiro B 1982 Phys. Rev. Lett. 48823

[7] Xiong G, Wang S D, Niu Q, Tian D C and Wang X R 2001 Phys. Rev. Lett. 87216802

[8] Chalker J T and Coddington P D 1988 J. Phys. C: Solid State Phys. 212665

[9] Abrahams E, Anderson P W, Licciardello D C and Ramakrishnan T V 1979 Phys. Rev. Lett. 42673

[10] Eilmes A, Römer R A and Schreiber M 1998 Eur. Phys. J. B 129

[11] Chan W L, Wang X R and Xie X C 1996 Phys. Rev. B 5411213

Xie X C, Wang X R and Liu D Z 1998 Phys. Rev. Lett. 803563

[12] Brouwer P W, Mudry C, Simons B D and Altland A 1998 Phys. Rev. Lett. 81862 